\title{
Discrete Filippov-Type Stability for One-Sided Lipschitzian Difference Inclusions
}

\author{
Robert Baier* and Elza Farkhi ${ }^{\dagger}$
}

30. August 2017

\begin{abstract}
We state and prove Filippov-type stability theorems for discrete difference inclusions obtained by the Euler discretization of a differential inclusion with perturbations in the set of initial points, in the right-hand side and in the state variable. We study the cases in which the right-hand side of the inclusion is not necessarily Lipschitz, but satisfies a weaker one-sided Lipschitz (OSL) or strengthened one-sided Lipschitz (SOSL) condition. The obtained estimates imply stability of the discrete solutions for infinite number of fixed time steps if the OSL constant is negative and the perturbations are bounded in certain norms. We show a better order of stability for SOSL right-hand sides and apply our theorems to estimate the distance from the solutions of other difference methods, as for the implicit Euler scheme to the set of solutions of the Euler scheme. We also prove a discrete relaxation stability theorem for the considered difference inclusion, which also extends a theorem of G. Grammel (2003) from the class of Lipschitz maps to the wider class of OSL ones.
\end{abstract}

Keywords: one-sided Lipschitz condition, strengthened one-sided Lipschitz condition, set-valued Euler's method, differential inclusions

Mathematics Subject Classifications (2010): 34A60, 47H05, 39A30, 54C60

\section{Introduction}

We regard the differential inclusion

$$
\left.\dot{x}(t) \in F(x(t)) \subset \mathbb{R}^{n} \quad \text { (a.e. } t \in I:=\left[t_{0}, T\right]\right), \quad x\left(t_{0}\right)=x^{0} \in X_{0}
$$

and its (set-valued) Euler discretization

$$
\eta^{j+1} \in \eta^{j}+h F\left(\eta^{j}\right), \quad j=0,1, \ldots, N-1, \quad \eta^{0}=x^{0} \in X_{0},
$$

where the initial set $X_{0} \subset \mathbb{R}^{n}$ is compact and nonempty, the step size is given by $h:=\frac{T-t_{0}}{N}$ for some $N \in \mathbb{N}$ and the grid points $t_{j}:=t_{0}+j h, j=0,1, \ldots, N$, form a grid $\mathcal{G}_{h}$ and a partition of $I$ in $N$ subintervals $I_{j}:=\left[t_{j}, t_{j+1}\right], j=0, \ldots, N-1$. For the sake of simplicity we consider

\footnotetext{
${ }^{*}$ Chair of Applied Mathematics, University of Bayreuth, 95440 Bayreuth, Germany, robert.baier@uni-bayreuth.de

${ }^{\dagger}$ School of Mathematical Sciences, Sackler Faculty of Exact Sciences, Tel Aviv University, 69978 Tel Aviv, Israel, elza@post.tau.ac.il
} 
here the autonomous case, although the results may be reformulated also for maps $F$ depending additionally on the time $t$.

We denote by $\mathcal{S}$ the set of solutions of (1) restricted to the grid $\mathcal{G}_{h}$ and by $\mathcal{S}_{h}$ the set of the solutions of (2). These sets are considered in the space of grid functions $\eta_{h}:=\left\{\eta^{j}\right\}_{j=0}^{N}$ with the usual Euclidean norm (see below).

In the classical Filippov Theorem [20] it is supposed that the map $F$ is Lipschitz in the state variable and existence and exponential Lipschitz stability of the set of solutions of (1) with respect to perturbations in the initial condition and the right-hand side is derived. The perturbed inclusion studied by Filippov in [20] is

$$
\dot{y}(t) \in F(y(t))+\bar{\varepsilon}(t) \quad(\text { a.e. } t \in I), \quad y\left(t_{0}\right)=y^{0} \in X_{0}
$$

with $\bar{\varepsilon}(t) \in \mathbb{R}^{n}$. For Lipschiz continuous multifunction $F$, the same stability rate as for the perturbations $\bar{\varepsilon}(t)$ in (3), called here 'outer' (set) perturbations, holds also for the inclusion with 'inner' (state) perturbations

$$
\dot{y}(t) \in F(y(t)+\bar{\delta}(t)), \quad(\text { a.e. } t \in I), \quad y\left(t_{0}\right)=y^{0} \in X_{0},
$$

where $\bar{\delta}(t) \in \mathbb{R}^{n}$. Removing the Lipschitz continuity usually leads to the loss of stability with respect to these perturbations. Fortunately, if the map $F$ is one-sided Lipschitz (OSL), the stability in the problem with inner and outer perturbation

$$
\dot{y}(t) \in F(y(t)+\bar{\delta}(t))+\bar{\varepsilon}(t) \quad(\text { a.e. } t \in I), \quad y\left(t_{0}\right)=y^{0} \in X_{0},
$$

is preserved, possibly in a weaker form [15, 16].

The OSL condition for single-valued functions $f: \mathbb{R}^{n} \rightarrow \mathbb{R}^{n}$ with constant $\mu \in \mathbb{R}$,

$$
\langle x-y, f(x)-f(y)\rangle \leq \mu|x-y|^{2} \quad\left(x, y \in \mathbb{R}^{n}\right),
$$

is known in numerical analysis (see e.g., [11, 3] and in [23, Sec. IV.12]), where $|\cdot|$ denotes the usual Euclidean norm in $\mathbb{R}^{n}$. In Hilbert and Banach spaces this concept was already known under the name dissipative respectively monotonic/accretive operators (see e.g., [34, 7, 8, 37]).

Here are the definitions of the two one-sided Lipschitz properties for set-valued maps investigated here.

Definition 1.1 ([15]) A set-valued map $F: \mathbb{R}^{n} \Rightarrow \mathbb{R}^{n}$ is called one-sided Lipschitz (OSL) with (OSL) constant $\mu \in \mathbb{R}$, i.e., for all $x, y \in \mathbb{R}^{n}$ and all $\xi \in F(x)$ there exists $\zeta \in F(y)$ with

$$
\langle x-y, \xi-\zeta\rangle \leq \mu|x-y|^{2} .
$$

For set-valued maps the OSL condition was first introduced in a stronger (uniform) form by KastnerMaresch and Lempio in [26, 29], and in a weaker (relaxed) abstract form in Banach spaces by Donchev (and Ivanov) [12, 18]. The condition of [26, 29], called here uniform one-sided Lipschitz (UOSL), requires that (7) is satisfied for all $x, y, \xi \in F(x), \zeta \in F(y)$. This condition implies uniqueness of the solution of (1) and allows convergence order 1 for 1 d problems ([29]) or, provided that the solution is piecewise smooth, for implicit Runge-Kutta methods with special stability properties $([26,27])$.

In [15] the most used explicit form of the OSL condition for set-valued maps in $\mathbb{R}^{n}$ was coined and the Filippov theorem [20] (with outer perturbations) was extended to the case of OSL righthand side of the inclusion. In [16] a more general Filippov theorem is proved for the inclusion (5) 
with OSL right-hand side and with both outer and inner perturbations. Then, Hölder one half rate of stability with respect to the inner perturbations is obtained. This result is applied there to obtain order of convergence $\mathcal{O}(\sqrt{h})$ for the Hausdorff distance between the sets of solutions of (1) and (2). The same order appears first in [33] for an OSL map $F(\cdot)$. Various generalizations of the OSL condition and of this important theorem may be found in [17]. We also refer the reader to the overview papers on OSL [33, 13, 14, 5].

Definition 1.2 ([33]) A set-valued map $F: \mathbb{R}^{n} \Rightarrow \mathbb{R}^{n}$ satisfies the strengthened one-sided Lipschitz (SOSL) condition with a (SOSL) constant $\mu \in \mathbb{R}$, if for all $x, y \in \mathbb{R}^{n}$ and all $\xi \in F(x)$ there exists $\zeta \in F(y)$ such that whenever $x_{i}>y_{i}$ for some $i \in\{1, \ldots, n\}$ we have the inequality

$$
\xi_{i}-\zeta_{i} \leq \mu|x-y|_{\infty}
$$

and whenever $x_{i}<y_{i}$ for some $i=1, \ldots, n$ we have the inequality

$$
\zeta_{i}-\xi_{i} \leq \mu|x-y|_{\infty}
$$

Here, $|\cdot|_{\infty}$ is the maximum norm and $z_{i}$ denotes the $i$-th coordinate of a vector $z:=\left(z_{1}, \ldots, z_{n}\right)^{\top} \in$ $\mathbb{R}^{n}$.

The maximum norm in (8)-(9) can be replaced by another vector norm, although it is a rather natural choice here. In [33, $n$ SOSL constants $\mu_{i} \in \mathbb{R}$ were introduced separately for each coordinate. Here, we use $\mu:=\max _{i=1, \ldots, n} \mu_{i}$ for simplicity.

In [30, 31] the uniform version of the latter condition requires that (8) holds for all $\zeta \in F(y)$ with $x_{i}>y_{i}$ (we call this version S-UOSL as in [5]). Due to the symmetry for $\xi \in F(x)$ and $\zeta \in F(y), 9$ is automatically fulfilled.

The strengthened one-sided Lipschitz condition (SOSL) essentially requires the OSL condition for each coordinate (in a given basis). Although it is stronger than the OSL condition, it does not imply continuity, but provides better stability than the OSL condition. It appears first in [30, 31] in an uniform form (S-UOSL, analogous to the UOSL condition). First order convergence of the Euler scheme for differential inclusions is derived for the S-UOSL right-hand side in [31, Sec. 4]. Lempio and Veliov formulated in [33] the weaker form as stated in Definition 1.2, analoguous to Definition 1.1, and proved that it ensures the first order convergence of the Euler scheme. The SOSL condition is stronger than the OSL condition and it has some interesting consequences which are not proved for general OSL maps as the order convergence $\mathcal{O}(h)$ of the Euler scheme instead of $\mathcal{O}(\sqrt{h})$ known for OSL maps. Also, the local existence of solutions of the differential inclusion (1) is shown in [19], provided the negation $-F$ is SOSL with zero constant. The latter property of the negation $-F$ defines a special type of monotonicity of $F$.

Here we prove a Filippov-type stability theorem for the solutions of a discrete inclusion of the form (2) with perturbations in the right-hand side, both in the state and the set, for OSL and SOSL maps and present some applications. Similarly to the 'continuous' Filippov-type theorem for OSL map $F$ [16], we show in the case of an OSL mapping $F$ stability of the discrete solution set which is of order one half with respect to inner and outer perturbations and with respect to the time step $h$. For infinite time interval, we obtain stability (boundedness) of the discrete solutions if the OSL constant is negative. In the case of OSL map $F$ we show first order of stability with respect to all perturbations and $h$. We apply these results to study the rate of convergence of the implicit Euler scheme in [6] for OSL (not necessarily continuous) maps $F$. In particular, we show that the iterates of the implicit Euler method are $\mathcal{O}(\sqrt{h})$-close to some iterates of the explicit one 
and even $\mathcal{O}(h)$-close for SOSL maps. An important possible application of the discrete Filippovtime theorems, together with the 'continuous' ones is to derive convergence rate of various discrete approximations of differential inclusions. Such discrete approximations like set-valued Euler and Runge-Kutta methods are studied in [40, 41, 45, and may be useful also for investigation of discrete approximations of control systems. Detailed analysis of such discretizations may be found in [42, 43, 39, 44, 24].

Let us remark that in [9, Proposition 2.2.3] a discrete Filippov-type theorem is proved in the Lipschitz case for the explicit Euler scheme and outer perturbations. In [6, Theorem 14] another discrete Filippov theorem is proved for the implicit Euler method and for outer perturbations in the case of time-dependent, jointly continuous and one-sided Lipschitz right-hand side.

We note that a discrete Filippov-type theorem can be deduced indirectly by continuous one [16], and the approximation estimate of the continuous trajectories by the discrete ones [15, 16]. We prefer the direct proofs to obtain more precise approximation estimates. The presented discrete Filippov theorems may be useful for investigation of the stability also for discrete systems obtained by one-step set-valued Runge-Kutta methods or some multistep methods as the leap-frog scheme, as well as for infinite time behavior, in particular in the case of negative OSL constant.

The paper is organized as follows. The next section contains some preliminaries and the basic assumptions. In Section 3 we prove the stability in the case of OSL mappings. The case of SOSL maps is discussed in Section 4. The applications are given in the last subsection of each section.

\section{Problem and Preliminaries}

In this section the notation and some preliminary results used further in the text are stated. We also present the problem formulation, the continuous differential inclusion and its discretization, the discrete Euler iterates.

\subsection{Preliminaries}

We denote by $\mathbb{R}_{+}:=\{x \in \mathbb{R} \mid x \geq 0\}$ and vectors in $\mathbb{R}^{n}$ by $x:=\left(x_{1}, x_{2}, \ldots, x_{n}\right)^{\top} \in \mathbb{R}^{n}$. The closed unit ball in $\mathbb{R}^{n}$ is denoted by $B_{1}(0)$, the usual scalar product of two vectors $x, y \in \mathbb{R}^{n}$ is denoted by $\langle x, y\rangle$. The corresponding Euclidean norm is denoted by $|x|_{2}$ or by $|x|$ for brevity, while the sum norm and the maximum norm of a vector $x \in \mathbb{R}^{n}$ are denoted by $|x|_{1}:=\sum_{i=1}^{n}\left|x_{i}\right|$ and $|x|_{\infty}:=\max _{1 \leq i \leq n}\left|x_{i}\right|$. For a real number $\mu$ we denote $\mu_{+}:=\max \{0, \mu\}$.

We denote by $\mathcal{K}\left(\mathbb{R}^{n}\right)$ the set of compact, nonempty subsets of $\mathbb{R}^{n}$. The Hausdorff distance between two sets $X, Y \in \mathcal{K}\left(\mathbb{R}^{n}\right)$ is

$$
\mathrm{d}_{\mathrm{H}}(X, Y):=\max \{\operatorname{dist}(X, Y), \operatorname{dist}(Y, X)\},
$$

where $\operatorname{dist}(X, Y):=\sup _{x \in X} \operatorname{dist}(x, Y)$ and the distance from a point to a set is $\operatorname{dist}(x, Y):=$ $\inf _{y \in Y}|x-y|$. The convex hull of a set $A$ is denoted by co $A$, the norm of a set is defined by $\|A\|:=\mathrm{d}_{\mathrm{H}}(A,\{0\})$.

For a $L_{p}$ function $f: I \rightarrow \mathbb{R}^{n}$ we denote $\|f\|_{L_{p}}$ as its $L_{p}$-norm and for a grid function $\eta_{h}=$ $\left\{\eta^{j}\right\}_{j=0}^{N-1}$ we define its discrete $L_{p}$-norm for $p \in\{1,2, \infty\}$, by

$$
\begin{aligned}
\left\|\eta_{h}\right\|_{1} & :=h \sum_{j=0}^{N-1}\left|\eta^{j}\right|, \quad\left\|\eta_{h}\right\|_{2}:=\sqrt{h \sum_{j=0}^{N-1}\left|\eta^{j}\right|^{2}}, \quad\left\|\eta_{h}\right\|_{\infty}:=\sup _{0 \leq j<N}\left|\eta^{j}\right|, \\
\left\lfloor\eta_{h}\right\rfloor_{\min , \mu} & :=\min \left\{\left\|\eta_{h}\right\|_{1}, \frac{1}{\sqrt{|\mu|}}\left\|\eta_{h}\right\|_{2}, \frac{1}{|\mu|}\left\|\eta_{h}\right\|_{\infty}\right\}
\end{aligned}
$$


We summarize the equivalence of the discrete $L_{p}$-norms for later reference.

Lemma 2.1 Let $h=\frac{T-t_{0}}{N}$ a given step size for $N \in \mathbb{N}$ and let $\eta_{h}=\left\{\eta_{h}^{j}\right\}_{j=0}^{N-1}$ be a grid function. Then,

$$
\begin{aligned}
\sqrt{h}\left\|\eta_{h}\right\|_{2} & \leq\left\|\eta_{h}\right\|_{1} \leq \sqrt{T-t_{0}}\left\|\eta_{h}\right\|_{2}, \\
h\left\|\eta_{h}\right\|_{\infty} & \leq\left\|\eta_{h}\right\|_{1} \leq\left(T-t_{0}\right)\left\|\eta_{h}\right\|_{\infty}, \\
\sqrt{h}\left\|\eta_{h}\right\|_{\infty} & \leq\left\|\eta_{h}\right\|_{2} \leq \sqrt{T-t_{0}}\left\|\eta_{h}\right\|_{\infty} .
\end{aligned}
$$

We denote the Hausdorff distance between two sets $\mathcal{S}, \widetilde{\mathcal{S}}$ of grid functions, using the $\|\cdot\|_{\infty}$ norm for the distance between the functions, by $\mathrm{d}_{\mathrm{H}}^{\infty}(\mathcal{S}, \widetilde{\mathcal{S}})$.

Next we present some notation and auxiliary inequalities used further in the text.

Denote $\overline{1}_{h}:=\{1\}_{j=0}^{N-1}$ and set for $\gamma_{h}=\left\{\gamma_{k}\right\}_{k=0}^{N-1} \subset \mathbb{R}_{+}$

$$
g_{h}\left(\mu, j ; \gamma_{h}\right):=h \sum_{k=0}^{j-1}(1+\mu h)^{j-1-k} \gamma_{k}, \quad g_{h}(\mu, j):=g_{h}\left(\mu, j ; \overline{1}_{h}\right) .
$$

Note that $g_{h}\left(\mu, j ; C^{\prime} \gamma_{h}^{\prime}+C^{\prime \prime} \gamma_{h}^{\prime \prime}\right)=C^{\prime} g_{h}\left(\mu, j ; \gamma_{h}^{\prime}\right)+C^{\prime \prime} g_{h}\left(\mu, j ; \gamma_{h}^{\prime \prime}\right)$.

Remark 2.2 Recall the simple claim that if $s_{j} \in \mathbb{R}, j=0,1, \ldots, k$, satisfy

$$
s_{k+1} \leq a \cdot s_{k}+\beta_{k}, \quad a \geq 0, \quad k=0,1, \ldots, j-1,
$$

then $s_{j} \leq a^{j} \cdot s_{0}+\sum_{k=0}^{j-1} a^{j-1+k} \beta_{k}$. Thus, for $a=1+\mu h$ and $\beta_{k}=h \gamma_{k}, k=0, \ldots N-1$, we get

$$
s_{j} \leq(1+\mu h)^{j} s_{0}+g_{h}\left(\mu, j ; \gamma_{h}\right), \quad j=0,1, \ldots, N-1 .
$$

We now estimate $g_{h}\left(\mu, j ; \gamma_{h}\right)$ and $g_{h}(\mu, j)$.

Lemma 2.3 Let $\mu \in \mathbb{R}, 1+\mu h>0, \gamma_{h}=\left\{\gamma_{k}\right\}_{k=0}^{N-1} \subset \mathbb{R}_{+}, t_{j}=t_{0}+j h, j=0, \ldots, N$. Then for $j=0, \ldots, N$,

$$
g_{h}\left(\mu, j ; \gamma_{h}\right) \leq e^{\mu_{+}\left(t_{j}-t_{0}\right)}\left\lfloor\left\lfloor\gamma_{h}\right\rfloor_{\min , \mu}\right.
$$

In particular,

$$
g_{h}(\mu, j) \leq e^{\mu_{+}\left(t_{j}-t_{0}\right)} \min \left\{t_{j}-t_{0}, \sqrt{\frac{t_{j}-t_{0}}{|\mu|}}, \frac{1}{|\mu|}\right\} .
$$

Proof: The case " $\mu=0$ " is trivial (we assume $\frac{1}{|\mu|}=\infty$ in the right-hand side).

Let $\mu \neq 0$. Note that since $e^{z} \geq 1+z$, we obtain for $\mu>0$ that

$$
(1+\mu h)^{j}-1 \leq(1+\mu h)^{j} \leq e^{\mu j h}=e^{\mu_{+} j h} .
$$

For $\mu<0$ we have

$$
\left|(1+\mu h)^{j}-1\right|=1-(1+\mu h)^{j}<1=e^{\mu_{+} j h} .
$$


Thus we obtain

$$
h \sum_{k=0}^{j-1}(1+\mu h)^{k}=h \frac{(1+\mu h)^{j}-1}{\mu h}=\frac{\left|(1+\mu h)^{j}-1\right|}{|\mu|} \leq \frac{1}{|\mu|} e^{\mu_{+} j h} .
$$

To show that $g_{h}\left(\mu, j ; \gamma_{h}\right) \leq e^{\mu_{+}\left(t_{j}-t_{0}\right)}\left\|\gamma_{h}\right\|_{1}$, we bound as in $18-19$ for $k \leq j$

$$
(1+\mu h)^{j-1-k} \leq e^{\mu_{+}(j-1-k) h} \leq e^{\mu_{+} j h}=e^{\mu_{+}\left(t_{j}-t_{0}\right)} .
$$

To show that $g_{h}\left(\mu, j ; \gamma_{h}\right) \leq \frac{1}{\sqrt{|\mu|}} e^{\mu_{+}\left(t_{j}-t_{0}\right)}\left\|\gamma_{h}\right\|_{2}$, we use the Hölder inequality

$h \sum_{k=0}^{j-1} \beta_{k} \gamma_{k} \leq\left(h \sum_{k=0}^{j-1}\left(\beta_{k}\right)^{2}\right)^{\frac{1}{2}}\left(h \sum_{k=0}^{j-1}\left(\gamma_{k}\right)^{2}\right)^{\frac{1}{2}}$ to get

$$
h \sum_{k=0}^{j-1}(1+\mu h)^{j-1-k} \gamma_{k} \leq\left(h \sum_{k=0}^{j-1}(1+\mu h)^{2 k}\right)^{\frac{1}{2}}\left\|\gamma_{h}\right\|_{2} .
$$

Then, using the formula for the geometric progression, the fact that $2+\mu h>1$ and $(18)-(19)$, we get

$$
\begin{aligned}
& \left(h \sum_{k=0}^{j-1}(1+\mu h)^{2 k}\right)^{\frac{1}{2}} \leq\left(h \frac{(1+\mu h)^{2 j}-1}{(1+\mu h)^{2}-1}\right)^{\frac{1}{2}}=\left(\frac{(1+\mu h)^{2 j}-1}{\mu(2+\mu h)}\right)^{\frac{1}{2}} \\
\leq & \left(\frac{\left|(1+\mu h)^{2 j}-1\right|}{|\mu| \cdot 1}\right)^{\frac{1}{2}} \leq\left(\frac{e^{\mu_{+} 2 j h}}{|\mu|}\right)^{\frac{1}{2}}=\frac{1}{\sqrt{|\mu|}} e^{\mu_{+}\left(t_{j}-t_{0}\right)} .
\end{aligned}
$$

The inequality $g_{h}\left(\mu, j ; \gamma_{h}\right) \leq \frac{1}{|\mu|}\left\|\gamma_{h}\right\|_{\infty}$ follows directly from 20 .

\subsection{Basic assumptions}

For definitions of notions as upper semi-continuity (usc) or measurability of set-valued maps and their properties which we do not define or formulate here in details, the reader may consult [2], [1] or [10].

The reachable set at time $T$ for the differential inclusion (1), starting from the set $X_{0}$, is denoted by $\mathcal{R}\left(T, t_{0}, X_{0}\right)$. For a given step-size $h:=\frac{T-t_{0}}{N}$ and grid points $t_{j}:=t_{0}+j h, j=0, \ldots, N$, let $\eta_{h}:=\left\{\eta^{j}\right\}_{j=0}^{N}, \eta^{j} \in \mathbb{R}^{n}$, be a discrete solution of the Euler inclusion (2). The discrete reachable set at time $T$ for the Euler inclusion (2), called $\mathcal{R}_{h}\left(T, t_{0}, X_{0}\right)$, is defined as the set of all end points $\eta^{N} \in \mathbb{R}^{n}$ of admissible grid functions starting from points of the set $X_{0}$.

Additionally we allow outer and inner perturbations of the discrete inclusion

$$
\eta^{j+1} \in \eta^{j}+h\left(F\left(\eta^{j}+\bar{\delta}^{j}\right)+\bar{\varepsilon}^{j}\right), \quad j=0, \ldots, N-1, \quad \eta^{0} \in X_{0},
$$

where the inner perturbations $\bar{\delta}_{h}:=\left\{\bar{\delta}^{j}\right\}_{j=0}^{N-1} \subset \mathbb{R}^{n}$ are uniformly bounded by a given constant $K_{\delta}$, while the outer ones $\bar{\varepsilon}_{h}:=\left\{\bar{\varepsilon}^{j}\right\}_{j=0}^{N-1} \subset \mathbb{R}^{n}$ are bounded in some discrete norm by a given constant $K_{\varepsilon}$.

For the rest of the paper we demand some of the following four assumptions:

(A1) $F(\cdot)$ has nonempty, compact images. 
$\left(\mathbf{A} 1^{\prime}\right) F(\cdot)$ has convex images.

(A2) There are constants $C_{B}, C_{F} \geq 0$ such that all solutions of $(22)$ satisfy

$$
\left\|\eta_{h}\right\|_{\infty} \leq C_{B} \quad \max _{0 \leq j \leq N}\left\|F\left(\eta^{j}\right)\right\| \leq C_{F} .
$$

At places where the solutions of (1) are involved we assume also

(A2') There exist solutions of (1) on $I$ and there are constants $C_{B}, C_{F} \geq 0$ such that all solutions of (1) satisfy

$$
\|x\|_{L_{\infty}} \leq C_{B}, \quad \sup _{t \in I}\|F(x(t))\| \leq C_{F} .
$$

Sufficient conditions for (A2') are discussed in the next remark.

Denote $S:=C_{B} B_{1}(0)$ such that $x(t) \in S$ for $t \in I$ and $\eta^{j} \in S$ for each $j=0, \ldots, N$.

(A3) $F(\cdot)$ is one-sided Lipschitz (OSL) with constant $\mu \in \mathbb{R}$, i.e., for all $x, y \in S$ and all $\xi \in F(x)$ there exists $\zeta \in F(y)$ with

$$
\langle x-y, \xi-\zeta\rangle \leq \mu|x-y|^{2}
$$

(A3') $F(\cdot)$ is strengthened one-sided Lipschitz (SOSL) with a constant $\mu \in \mathbb{R}$, i.e., for all $x, y \in S$ and all $\xi \in F(x)$ there exists $\zeta \in F(y)$ such that if $x_{i}>y_{i}$ we have the inequality

$$
\xi_{i}-\zeta_{i} \leq \mu|x-y|_{\infty}
$$

and whenever $x_{i}<y_{i}$,

$$
\zeta_{i}-\xi_{i} \leq \mu|x-y|_{\infty}
$$

Additionally, we sometimes require the assumption

(A0) $F: \mathbb{R}^{n} \Rightarrow \mathbb{R}^{n}$ is upper semi-continuous (usc), i.e., for all $x \in S$ and all $\varepsilon>0$ there exists $\delta>0$ such that for all $y \in \mathbb{R}^{n}$ with $|x-y| \leq \delta, F(y) \subset F(x)+\varepsilon B_{1}(0)$.

Remark 2.4 The assumption (A2') can be guaranteed by assuming a linear growth condition, i.e., $\|F(t, x)\| \leq C(1+|x|)$ for all $x \in \mathbb{R}^{n}$ (see [15, Lemmas 3.1 and 4.1], [4, Lemma 2.6]), or by the weaker assumption of boundedness of $F(\cdot)$ on bounded sets together with the OSL condition on $\mathbb{R}^{n}$ (see [16, Lemma 3.2 and Remark 3.1]). To guarantee the existence in (A2') one can require additionally e.g., (A0), (A1) and (A1)', see [10, Ch. 2]. For simplicity we do not formulate the weakest possible assumptions.

\section{Discrete Filippov-Type Theorems for One-Sided Lipschitz Maps}

Here we discuss the stability with respect to inner and outer perturbations separately, for reader's convenience. 


\section{$3.1 \quad$ Outer perturbations}

We consider perturbed initial values and outer perturbations. A discrete counterpart of the continuous Filippov theorem for OSL maps in [16] with $\bar{\delta}(t) \equiv 0$ is obtained.

Proposition 3.1 Let the assumptions (A1)-(A3) and $1+2 \mu h>0$ be satisfied. Consider $\bar{\varepsilon}_{h}=$ $\left\{\bar{\varepsilon}^{j}\right\}_{j=0}^{N-1} \subset \mathbb{R}^{n}$ with $\left\|\bar{\varepsilon}_{h}\right\|_{\infty} \leq K_{\varepsilon}$ and let $\left\{y^{j}\right\}_{j=0}^{N}$ be a discrete solution of the perturbed inclusion

$$
y^{j+1} \in y^{j}+h\left(F\left(y^{j}\right)+\bar{\varepsilon}^{j}\right), \quad j=0, \ldots, N-1, \quad y^{0} \in X_{0} \text { be given } .
$$

Then there exists a discrete solution $\left\{x^{j}\right\}_{j=0}^{N}$ of (2) with

$$
\begin{aligned}
& \left|y^{j+1}-x^{j+1}\right|^{2} \leq(1+2 \mu h)\left|y^{j}-x^{j}\right|^{2}+4 C_{B} \varepsilon_{j} h+2\left(\varepsilon_{j}^{2}+4 C_{F}^{2}\right) h^{2}, \\
& \left|y^{j}-x^{j}\right| \leq(\sqrt{1+2 \mu h})^{j}\left|y^{0}-x^{0}\right|+C_{1} \sqrt{g_{h}\left(2 \mu, j, \varepsilon_{h}\right)}+C_{2} \sqrt{g_{h}(2 \mu, j)} \sqrt{h}
\end{aligned}
$$

where $\varepsilon_{h}=\left\{\varepsilon_{j}\right\}_{j=0}^{N-1}, \varepsilon_{j}=\left|\bar{\varepsilon}^{j}\right|, \quad C_{1}=\sqrt{4 C_{B}+2 C_{\varepsilon}}, C_{2}=2 \sqrt{2} C_{F}, C_{\varepsilon}=\left(T-t_{0}\right) K_{\varepsilon}$.

Proof: By (A2), $y^{j} \in S$ for each $j=0, \ldots, N$. Given a solution $\left\{y^{j}\right\}_{j=0}^{N}$ of (23), there is $w^{j} \in F\left(y^{j}\right)$ with

$$
y^{j+1}=y^{j}+h\left(w^{j}+\bar{\varepsilon}^{j}\right), \quad j=0, \ldots, N-1 .
$$

Suppose the iterates $x^{k}$ are constructed for $0 \leq k \leq j$. By the OSL condition choose $v^{j} \in F\left(x^{j}\right)$ such that $\left\langle y^{j}-x^{j}, w^{j}-v^{j}\right\rangle \leq \mu\left|y^{j}-x^{j}\right|^{2}$. Then,

$$
\begin{aligned}
\left\langle y^{j}-x^{j}, w^{j}+\bar{\varepsilon}^{j}-v^{j}\right\rangle & =\left\langle y^{j}-x^{j}, w^{j}-v^{j}\right\rangle+\left\langle y^{j}-x^{j}, \bar{\varepsilon}^{j}\right\rangle \\
& \leq \mu\left|y^{j}-x^{j}\right|^{2}+\left|y^{j}-x^{j}\right| \cdot \varepsilon_{j} .
\end{aligned}
$$

We set $x^{j+1}:=x^{j}+h v^{j}$ which yields

$$
y^{j+1}-x^{j+1}=\left(y^{j}-x^{j}\right)+h\left(w^{j}+\bar{\varepsilon}^{j}-v^{j}\right) .
$$

Using the inequality $(a+b)^{2} \leq 2\left(a^{2}+b^{2}\right)$ and (A2), we get

$$
\left|w^{j}+\bar{\varepsilon}^{j}-v^{j}\right|^{2} \leq 2\left(\left|w^{j}-v^{j}\right|^{2}+\left|\bar{\varepsilon}^{j}\right|^{2}\right) \leq 8 C_{F}^{2}+2 \varepsilon_{j}^{2} .
$$

We use this inequality and (26) in the estimate of the norm difference:

$$
\begin{aligned}
\left|y^{j+1}-x^{j+1}\right|^{2} & =\left|y^{j}-x^{j}\right|^{2}+2 h\left\langle y^{j}-x^{j}, w^{j}+\bar{\varepsilon}^{j}-v^{j}\right\rangle+\left|w^{j}+\bar{\varepsilon}^{j}-v^{j}\right|^{2} h^{2} \\
& \leq(1+2 \mu h)\left|y^{j}-x^{j}\right|^{2}+2 \varepsilon_{j} h\left|y^{j}-x^{j}\right|+2 \varepsilon_{j}^{2} h^{2}+8 C_{F}^{2} h^{2}
\end{aligned}
$$

We apply (A2) to estimate the second term in the right-hand side,

$$
2 \varepsilon_{j} h\left|y^{j}-x^{j}\right| \leq 2 \varepsilon_{j} h\left(\left|y^{j}\right|+\left|x^{j}\right|\right) \leq 4 C_{B} \varepsilon_{j} h .
$$

The last two inequalities imply (24). Denoting $s_{j}:=\left|y^{j}-x^{j}\right|^{2}, \varepsilon_{h}^{2}:=\left\{\varepsilon_{j}^{2}\right\}_{j=0}^{N-1}$ and using (15), we obtain

$$
\begin{aligned}
s_{j} & \leq(1+2 \mu h)^{j} s_{0}+g_{h}\left(2 \mu, j ; 4 C_{B} \varepsilon_{h}+2 h \varepsilon_{h}^{2}+8 C_{F}^{2} h \overline{1}_{h}\right) \\
& =(1+2 \mu h)^{j} s_{0}+4 C_{B} g_{h}\left(2 \mu, j ; \varepsilon_{h}\right)+2 h g_{h}\left(2 \mu, j ; \varepsilon_{h}^{2}\right)+8 C_{F}^{2} h g_{h}(2 \mu, j)
\end{aligned}
$$


To simplify the estimate (27), we note that since by (A2) $y^{j}, y^{j+1}, F\left(y^{j}\right)$ are uniformly bounded, $h \varepsilon_{j}$ is uniformly bounded too. Let $C_{\varepsilon}$ be a bound of $h \varepsilon_{j}$, then

$$
g_{h}\left(2 \mu, j ; \varepsilon_{h}^{2}\right) \sqrt{h} \leq C_{\varepsilon} g_{h}\left(2 \mu, j ; \varepsilon_{h}\right)
$$

and $C_{\varepsilon} \leq 2 C_{B}+h C_{F}$. Applied to (27), this yields

$$
s_{j} \leq(1+2 \mu h)^{j} s_{0}+\left(4 C_{B}+2 C_{\varepsilon}\right) g_{h}\left(2 \mu, j ; \varepsilon_{h}\right)+8 C_{F}^{2} h g_{h}(2 \mu, j) .
$$

Taking the square root we obtain the following estimate

$$
\left|y^{j}-x^{j}\right| \leq(\sqrt{1+2 \mu h})^{j}\left|y^{0}-x^{0}\right|+C_{1} \sqrt{g_{h}\left(2 \mu, j ; \varepsilon_{h}\right)}+C_{2} \sqrt{g_{h}(2 \mu, j)} \sqrt{h}
$$

with the constants $C_{1}:=\sqrt{4 C_{B}+2 C_{\varepsilon}}, C_{2}:=2 \sqrt{2} C_{F}$.

Then, applying Lemma 2.3 to 25 we get

Theorem 3.2 (discrete Filippov theorem with outer perturbations) Assuming the conditions of Proposition 3.1 with a step size $h=\frac{T-t_{0}}{N}$ such that $1+2 \mu h>0$.

Then, for a discrete solution $y_{h}=\left\{y^{j}\right\}_{j=0}^{N}$ of the perturbed Euler inclusion 23, there exists a discrete solution $x_{h}=\left\{x^{j}\right\}_{j=0}^{N}$ of the Euler inclusion (2) with

$$
\begin{aligned}
\left|y^{j}-x^{j}\right| \leq & (\sqrt{1+2 \mu h})^{j}\left|y^{0}-x^{0}\right| \\
& +e^{\mu_{+}\left(t_{j}-t_{0}\right)}\left\{C_{1} \sqrt{\left\lfloor\varepsilon_{h}\right\rfloor_{\min , 2 \mu}}+C_{2} \sqrt{\left\lfloor\left\lfloor\overline{1}_{h}\right\rfloor\right\rfloor_{\min , 2 \mu}} \sqrt{h}\right\}
\end{aligned}
$$

with $\varepsilon_{h}=\left\{\varepsilon_{j}\right\}_{j=0}^{N-1}, \varepsilon_{j}=\left|\bar{\varepsilon}^{j}\right|, \quad C_{1}:=\sqrt{4 C_{B}+2 C_{\varepsilon}}, \quad C_{2}:=2 \sqrt{2} C_{F}, \quad C_{\varepsilon}:=\left(T-t_{0}\right) K_{\varepsilon}$.

Remark 3.3 A similar but coarser estimate than (25) (with the norm $\left\|\varepsilon_{h}\right\|_{1}$ and bigger constants) follows in an indirect way, applying the continuous Filippov theorem for the OSL case (Theorem 3.1 in [16]) and the error estimate for the Euler approximation (Theorem 4.1 for convex-valued maps in the same paper). In the theorem above we provide a direct proof with a refined estimate.

Note that $g_{h}\left(2 \mu, j ; \varepsilon_{h}\right)$ in (25) corresponds to the Riemann sum of the integral error term $e^{2 \mu(t-\cdot)} \varepsilon(\cdot)$ in the above cited continuous-time Filippov theorem in [16].

Corollary 3.4 If we additionally assume in Theorem 3.2 that

$$
\left\lfloor\varepsilon_{h}\right\rfloor_{\min , \mu} \leq C_{\alpha} h^{\alpha}
$$

with $\alpha>0$, then, since $1+z \leq e^{z}$, we may find a constant $C$ such that

$$
\left|y^{j}-x^{j}\right| \leq e^{\mu\left(t_{j}-t_{0}\right)}\left|y^{0}-x^{0}\right|+C h^{\frac{1}{2} \min \{\alpha, 1\}}
$$

with $C$ depending on $\mu$ and, for $\mu=0$ additionally, on $t_{j}-t_{0}$.

To obtain stability estimate for an infinite time (when $N \rightarrow \infty, h>0$ is fixed), we define the infinite-time discrete norms for $\varepsilon_{h}^{\infty}:=\left\{\varepsilon_{j}\right\}_{j=0}^{\infty}$ as in 10 11) by replacing $N-1, N$ by $\infty$ and suppose that $\mu<0$. Then we get from (30) a discrete version of the stability result in [16, Corollary 3.2], [21]. 
Theorem 3.5 Assuming the conditions of Proposition 3.1 on $I=\left[t_{0}, \infty\right)$, especially $\left\|\bar{\varepsilon}_{h}^{\infty}\right\|_{\infty} \leq K_{\varepsilon}$, and let the OSL constant $\mu<0$. For a fixed step size $h>0$ with $1+2 \mu h>0$ we consider infinitely many steps with the Euler method.

Then, for a discrete solution of the perturbed Euler inclusion $y_{h}^{\infty}=\left\{y^{j}\right\}_{j=0}^{\infty}$ there exists a discrete solution $x_{h}^{\infty}=\left\{x^{j}\right\}_{j=0}^{\infty}$ of the Euler inclusion (2) with

$$
\left|y^{j}-x^{j}\right| \leq e^{\mu\left(t_{j}-t_{0}\right)}\left|y^{0}-x^{0}\right|+C_{1} \sqrt{\left\lfloor\left\lfloor\left\{\varepsilon_{\nu}\right\}_{\nu=0}^{j-1}\right\rfloor \min , \mu\right.}+C_{2} \frac{1}{\sqrt{|\mu|}} \sqrt{h} .
$$

The Hausdorff distance between the original and the perturbed reachable sets is

$$
\limsup _{j \rightarrow \infty} \mathrm{d}_{\mathrm{H}}\left(\mathcal{R}_{h}\left(t_{j}, t_{0}, X_{0}\right), \mathcal{R}_{h}^{\varepsilon_{h}}\left(t_{j}, t_{0}, X_{0}\right)\right) \leq C_{1} \sqrt{\left\lfloor\left\lfloor\varepsilon_{h}^{\infty}\right\rfloor_{\min , \mu}\right.}+C_{2} \frac{1}{\sqrt{|\mu|}} \sqrt{h}
$$

Thus, if $\left\lfloor\varepsilon_{h}^{\infty}\right\rfloor_{\min , \mu}=\mathcal{O}(h)$, in particular if $\sum_{j=0}^{\infty} \varepsilon_{j}<\infty$, the estimate is

$$
\limsup _{j \rightarrow \infty} \mathrm{d}_{\mathrm{H}}\left(\mathcal{R}_{h}\left(t_{j}, t_{0}, X_{0}\right), \mathcal{R}_{h}^{\varepsilon_{h}}\left(t_{j}, t_{0}, X_{0}\right)\right)=\mathcal{O}(\sqrt{h}) .
$$

\subsection{Inner perturbations}

Here, the right-hand side contains only inner perturbation of the state variable.

Theorem 3.6 (discrete Filippov theorem with inner perturbations) Let the assumptions (A1)-(A3) and $1+2 \mu h>0$ be satisfied and consider a grid function $\bar{\delta}_{h}:=\left\{\bar{\delta}^{j}\right\}_{j=0}^{N} \subset \mathbb{R}^{n}$ with $\delta_{j}:=\left|\bar{\delta}^{j}\right|, j=0, \ldots, N, \delta_{h}:=\left\{\delta_{j}\right\}_{j=0}^{N}$ and $\left\|\bar{\delta}_{h}\right\|_{\infty} \leq K_{\delta}$. Let $\left\{y^{j}\right\}_{j=0}^{N}$ be a discrete solution of the perturbed Euler inclusion

$$
y^{j+1} \in y^{j}+h F\left(y^{j}+\bar{\delta}^{j}\right) \quad(j=0, \ldots, N-1), \quad y^{0} \in X_{0} \quad \text { given } .
$$

Then for every $x^{0} \in X_{0}$ there exists a discrete solution $\left\{x^{j}\right\}_{j=0}^{N}$ of (2) with

$$
\begin{aligned}
\left|y^{j}-x^{j}\right| \leq & (\sqrt{1+2 \mu h})^{j}\left|y^{0}-x^{0}\right|+C_{1} \sqrt{g_{h}\left(2 \mu, j, \delta_{h}\right)}+C_{2} \sqrt{g_{h}(2 \mu, j)} \sqrt{h} \\
\left|y^{j}-x^{j}\right| \leq & (\sqrt{1+2 \mu h})^{j}\left|y^{0}-x^{0}\right| \\
& +e^{\mu_{+}\left(t_{j}-t_{0}\right)}\left\{C_{1} \sqrt{\left\lfloor\delta_{h}\right\rfloor_{\min , 2 \mu}}+C_{2} \sqrt{\left\lfloor\left\lfloor\overline{1}_{h}\right\rfloor\right\rfloor_{\min , 2 \mu}} \sqrt{h}\right\}
\end{aligned}
$$

for $j=0, \ldots, N$ with constants $C_{1}:=2 \sqrt{C_{F}+\left(2 C_{B}+\frac{1}{2} K_{\delta}\right)|\mu|}$, and $C_{2}:=2 C_{F}$.

Proof: Assume that we have constructed the sequence $\left\{x^{k}\right\}_{k}$ up to the index $j$. Let $y^{j+1}=$ $y^{j}+h w^{j}, w^{j} \in F\left(y^{j}+\bar{\delta}^{j}\right)$. The OSL condition assures the existence of $v^{j} \in F\left(x^{j}\right)$ such that

$$
\left\langle\left(y^{j}+\bar{\delta}^{j}\right)-x^{j}, w^{j}-v^{j}\right\rangle \leq \mu\left|\left(y^{j}+\bar{\delta}^{j}\right)-x^{j}\right|^{2}
$$

and we define $x^{j+1}$ by

$$
x^{j+1}:=x^{j}+h v^{j} .
$$


By assumption (A2) the sequence $\left\{v^{j}\right\}_{j=0}^{N-1},\left\{w^{j}\right\}_{j=0}^{N-1}$ are uniformly bounded by $C_{F}$. Hence,

$$
\begin{aligned}
\left|y^{j+1}-x^{j+1}\right|^{2}= & \left|\left(y^{j}+h w^{j}\right)-\left(x^{j}+h v^{j}\right)\right|^{2}=\left|\left(y^{j}-x^{j}\right)+h\left(w^{j}-v^{j}\right)\right|^{2} \\
\leq & \left|y^{j}-x^{j}\right|^{2}+2 h\left\langle\left(y^{j}+\bar{\delta}^{j}\right)-x^{j}, w^{j}-v^{j}\right\rangle \\
& -2 h\left\langle\bar{\delta}^{j}, w^{j}-v^{j}\right\rangle+4 C_{F}^{2} h^{2} \\
\leq & \left|y^{j}-x^{j}\right|^{2}+2 \mu h\left|\left(y^{j}+\bar{\delta}^{j}\right)-x^{j}\right|^{2}+2 h\left|\bar{\delta}^{j}\right| \cdot\left|w^{j}-v^{j}\right|+4 C_{F}^{2} h^{2},
\end{aligned}
$$

since the difference $\left|w^{j}-v^{j}\right|$ can be bounded by $2 C_{F}$. We estimate

$$
\begin{aligned}
\mu\left|\left(y^{j}+\bar{\delta}^{j}\right)-x^{j}\right|^{2} & =\mu\left(\left|y^{j}-x^{j}\right|^{2}+2\left\langle y^{j}-x^{j}, \bar{\delta}^{j}\right\rangle+\left|\bar{\delta}^{j}\right|^{2}\right) \\
& \leq \mu\left|y^{j}-x^{j}\right|^{2}+2|\mu| \cdot\left|y^{j}-x^{j}\right| \cdot\left|\bar{\delta}^{j}\right|+\mu\left|\bar{\delta}^{j}\right|^{2} \\
& \leq \mu\left|y^{j}-x^{j}\right|^{2}+4 C_{B}|\mu| \delta_{j}+|\mu| \cdot \delta_{j}^{2} .
\end{aligned}
$$

The last inequalities and the bound $\delta_{j}^{2} \leq K_{\delta} \delta_{j}$ lead to

$$
\begin{aligned}
\left|y^{j+1}-x^{j+1}\right|^{2} \leq & \left|y^{j}-x^{j}\right|^{2}+2 \mu h\left|y^{j}-x^{j}\right|^{2}+2|\mu| h \delta_{j}^{2} \\
& +8 C_{B}|\mu| h \delta_{j}+4 C_{F} h \delta_{j}+4 C_{F}^{2} h^{2} \\
\leq & (1+2 \mu h)\left|y^{j}-x^{j}\right|^{2}+4\left(C_{F}+\left(2 C_{B}+\frac{1}{2} K_{\delta}\right)|\mu|\right) h \delta_{j}+4 C_{F}^{2} h^{2} .
\end{aligned}
$$

We set $\Delta^{j}:=\left|y^{j}-x^{j}\right|^{2}$ and as in Remark 2.2 get

$$
\begin{aligned}
\Delta^{j+1} & \leq(1+2 \mu h) \Delta^{j}+4\left(C_{F}+\left(2 C_{B}+\frac{1}{2} K_{\delta}\right)|\mu|\right) h \delta_{j}+4 C_{F}^{2} h^{2}, \\
\Delta^{j} & \leq(1+2 \mu h)^{j} \Delta^{0}+\sum_{k=0}^{j-1}(1+2 \mu h)^{j-1-k}\left(C_{1}^{2} h \delta_{j}+4 C_{F}^{2} h^{2}\right)
\end{aligned}
$$

Taking the square root yields

$$
\left|y^{j}-x^{j}\right| \leq(\sqrt{1+2 \mu h})^{j}\left|y^{0}-x^{0}\right|+C_{1} \sqrt{g_{h}\left(2 \mu, j, \delta_{h}\right)}+2 C_{F} \sqrt{g_{h}(2 \mu, j)} \sqrt{h} .
$$

We complete the proof applying Lemma 2.3 .

Corollary 3.7 Let all assumptions of Theorem 3.6 are fulfilled and let

$$
\left\lfloor\left\lfloor\delta_{h}\right\rfloor\right\rfloor_{\min , \mu} \leq C_{\alpha} h^{\alpha}
$$

be fulfilled for the inner perturbation.

Then, for each solution $\left\{y^{j}\right\}_{j=0}^{N}$ of the perturbed inclusion (32) there exists a discrete solution $\left\{x^{j}\right\}_{j=0}^{N}$ of 2 with

$$
\left|y^{j}-x^{j}\right| \leq e^{\mu\left(t_{j}-t_{0}\right)}\left|y^{0}-x^{0}\right|+\widetilde{C} e^{\mu+\left(T-t_{0}\right)} h^{\frac{1}{2} \min \{\alpha, 1\}},
$$

where the constant $\widetilde{C}$ may be easily estimated from $C_{1}, C_{2}$ in Theorem 3.6 and does not depend on the time length whenever $\mu \neq 0$. 


\subsection{Both perturbations and applications}

The general theorem for inner and outer perturbations may be obtained combining the last two theorems. In the estimate the square root of the discrete norms of the inner and outer perturbations as well as an error term $\mathcal{O}(\sqrt{h})$ will appear.

Theorem 3.8 (discrete Filippov theorem with both perturbations) Let the assumptions (A1)(A3) and $1+2 \mu h>0$ hold and consider grid functions $\bar{\delta}_{h}=\left\{\bar{\delta}^{j}\right\}_{j=0}^{N-1} \subset \mathbb{R}^{n}$ with $\delta_{j}:=\left|\bar{\delta}^{j}\right|$, $j=0, \ldots, N-1$, and $\bar{\varepsilon}_{h}=\left\{\bar{\varepsilon}^{j}\right\}_{j=0}^{N-1} \subset \mathbb{R}^{n}, \varepsilon_{j}:=\left|\bar{\varepsilon}^{j}\right|$ satisfying $\left\|\bar{\delta}_{h}\right\|_{\infty} \leq K_{\delta},\left\|\bar{\varepsilon}_{h}\right\|_{\infty} \leq K_{\varepsilon}$. Let $\left\{y^{j}\right\}_{j=0}^{N}$ be a discrete solution of the perturbed Euler inclusion

$$
y^{j+1} \in y^{j}+h\left(F\left(y^{j}+\bar{\delta}^{j}\right)+\bar{\varepsilon}^{j}\right), \quad j=0, \ldots, N-1, \quad y^{0} \in X_{0} \text { is given. }
$$

Then for every $x^{0} \in X_{0}$ there exists a discrete solution $\left\{x^{j}\right\}_{j=0}^{N}$ of (2) with

$$
\begin{aligned}
\left|y^{j}-x^{j}\right| \leq & (\sqrt{1+2 \mu h})^{j}\left|y^{0}-x^{0}\right|+C_{1} \sqrt{g_{h}\left(2 \mu, j, \delta_{h}\right)} \\
& +C_{2} \sqrt{g_{h}\left(2 \mu, j, \varepsilon_{h}\right)}+C_{3} \sqrt{g_{h}(2 \mu, j)} \sqrt{h}
\end{aligned}
$$

for $j=0, \ldots, N$ with constants $C_{1}:=2 \sqrt{C_{F}+\left(2 C_{B}+\frac{1}{2} K_{\delta}\right)|\mu|}, C_{2}:=\sqrt{4 C_{B}+2 C_{\varepsilon}}, C_{3}:=$ $(2+2 \sqrt{2}) C_{F}$ and $C_{\varepsilon}:=\left(T-t_{0}\right) K_{\varepsilon}$.

Next we study the distance between the iterates of the explicit and the implicit set-valued Euler's method. The following proposition shows that each iterate of the second is close to some iterate of the first one, and thus provides convergence results for the implicit method whenever the corresponding convergence result for the explicit method is known. A more elaborated study for continuous right-hand sides can be found in [6]. It is also shown in [6] that if $F$ is usc and $1-\mu h>0$, then the implicit inclusion (37) has a solution.

Proposition 3.9 Let the step size $h$ be so small that $h C_{F} \leq K_{\delta}, 1-\mu h>0$ and choose $x^{0} \in X_{0}$. In addition to (AO)-(A3), assume that (A1') is fulfilled and (A2) also holds for the implicit Euler method.

Then there is a constant $C$ such that for each implicit Euler iterate $\left\{y^{j}\right\}_{j=0}^{N}$ of

$$
y^{j+1} \in y^{j}+h F\left(y^{j+1}\right), \quad j=0,1, \ldots, N-1, \quad y^{0}=x^{0},
$$

there is an iterate $\left\{x^{j}\right\}_{j=0}^{N}$ of the explicit scheme (2) with

$$
\left|y^{j}-x^{j}\right| \leq C \sqrt{h}, \quad j=0, \ldots, N
$$

and the distance from the reachable set of (37) to the one of (2) satisfies

$$
\operatorname{dist}\left(\mathcal{R}_{h}^{i m p l}\left(T, t_{0}, X_{0}\right), \mathcal{R}_{h}\left(t_{j}, t_{0}, X_{0}\right)\right) \leq C \sqrt{h}
$$

Proof: The restriction on the step size $h$ and (A0), (A1'), (A3) guarantee the existence of iterates of the implicit Euler method by [6, Theorem 4].

Consider an iterate of the implicit scheme, i.e.,

$$
y^{j+1}=y^{j}+h w^{j+1}, \quad w^{j+1} \in F\left(y^{j+1}\right) .
$$


We can rewrite it as perturbed Euler iteration with $\bar{\delta}^{j}:=y^{j+1}-y^{j}$, since

$$
y^{j+1} \in y^{j}+h F\left(y^{j+1}\right), \quad F\left(y^{j+1}\right)=F\left(y^{j}+\bar{\delta}^{j}\right) .
$$

The inner perturbations are bounded by $\mathcal{O}(h)$, since iterates of both schemes (and hence velocities) are bounded:

$$
\left|\bar{\delta}^{j}\right|=\left|y^{j+1}-y^{j}\right|=h\left|w^{j+1}\right| \leq C_{F} h \leq K_{\delta}
$$

Corollary 3.7 for the explicit Euler can be applied so that

$$
\left|y^{j}-x^{j}\right| \leq \widetilde{C}_{1} \sqrt{\left\|\delta_{h}\right\|_{1}}+\widetilde{C}_{2} \sqrt{h}=\left(\widetilde{C}_{1} \sqrt{C_{F}}+\widetilde{C}_{2}\right) \sqrt{h} .
$$

Corollary 3.10 Let the assumptions of Proposition 3.9 here and of Theorem 4.1 in [16] be fulfilled. Then, there exists a constant $C$ with

$$
\operatorname{dist}\left(\mathcal{R}_{h}^{i m p l}\left(T, t_{0}, X_{0}\right), \mathcal{R}\left(T, t_{0}, X_{0}\right)\right) \leq C \sqrt{h} .
$$

Proof: Since $F$ is convex-valued, we can apply the convergence result for the explicit Euler in [16, Theorem 4.1] so that

$$
\mathrm{d}_{\mathrm{H}}\left(\mathcal{R}_{h}\left(T, t_{0}, X_{0}\right), \mathcal{R}\left(T, t_{0}, X_{0}\right)\right) \leq C \sqrt{h} .
$$

The rest follows by Proposition 3.9 and the triangle inequality.

Remark 3.11 Similarly, $\mathcal{O}(\sqrt{h})$-estimates for other Runge-Kutta methods may be obtained applying Corollary 3.10 if $F$ is OSL. As one example we mention the improved Euler scheme in [30]

$$
x^{j+1} \in x^{j}+h F\left(x^{j}+\frac{h}{2} v^{j}\right), \quad v^{j} \in F\left(x^{j}\right) .
$$

Here, $\left|\bar{\delta}^{j}\right|=\frac{h}{2}\left|v^{j}\right| \leq \frac{C_{F}}{2} h$ and the order of the distance is $\mathcal{O}(\sqrt{h})$.

Remark 3.12 We can formulate Proposition 3.9 and Corollary 3.10 with the same assumptions and the Lipschitz condition replacing the OSL one provided that (A1') also holds. Then, explicit and implicit Euler iterates can be found that are $\mathcal{O}(h)$-close by applying the discrete Filippov theorem for the explicit Euler in [9, Proposition 2.2.3] as well as the corresponding Filippov theorem for the implicit Euler in [6, Theorem 14]. Therefore, the convergence of the implicit Euler is the same as for the explicit one, i.e., $\mathcal{O}(h)$ on a finite time interval. But, the OSL condition with a negative constant provides contractivity of the reachable set mapping and (exponential) stability at an infinite time interval.

Both mentioned convergence results for the implicit Euler method for OSL maps with additional continuous right-hand sides do not deliver the preferrable stability results for $\mu<0$ as stated in [6]. 


\subsection{Discrete relaxation stability theorem}

Consider the (set-valued) Euler discrete inclusion

$$
\eta^{j+1} \in \eta^{j}+h F\left(\eta^{j}\right), \quad j=0,1, \ldots, N-1, \quad \eta^{0}=x^{0} \in X_{0},
$$

and its convexified counterpart

$$
\eta^{j+1} \in \eta^{j}+h \operatorname{co} F\left(\eta^{j}\right), \quad j=0,1, \ldots, N-1, \quad \eta^{0}=x^{0} .
$$

In [22], an estimate of order $\mathcal{O}(\sqrt{h})$ is obtained for the Hausdorff distance between the solutions sets of the relaxed differential inclusion (with co $F$ at the right-hand side in (1)) and the Euler difference inclusion (38).

We denote by $\mathcal{S}_{h}$ the set of solutions of $(38)$ and by $\mathcal{S}_{h}^{c o}$ the set of solutions of (39). Here, these solutions are considered in the space of grid functions $\eta_{h}=\left\{\eta^{j}\right\}_{j=0}^{N}$ and are studied under the weaker OSL condition.

Theorem 3.13 (discrete relaxation stability) Let the assumptions (A1)-(A3) hold.

Then, there is a constant $C$ such that

$$
\mathrm{d}_{\mathrm{H}}\left(\mathcal{S}_{h}, \mathcal{S}_{h}^{c o}\right) \leq C \sqrt{h} .
$$

Proof: For a solution $\left\{y^{j}\right\}_{j=0}^{N}$ of $(39)$ and all $0 \leq j \leq N-1$ there is $w^{j} \in \operatorname{co} F\left(y^{j}\right)$ with

$$
y^{j+1}=y^{j}+h w^{j}, \quad j=0, \ldots, N-1 .
$$

We construct a solution $\left\{x^{j}\right\}_{j=0}^{N}$ of 38 which is at the required distance from $\left(y^{j}\right)_{j=0}^{N}$. Suppose $x^{k}$ are constructed for $0 \leq k \leq j$. We recall that since $F(x)$ is OSL, then also the map co $F(x)$ is OSL with the same constant (this can be easily verified by the definition).

By the OSL condition there is $\widetilde{v}^{j} \in \operatorname{co} F\left(x^{j}\right)$ such that

$$
\left\langle y^{j}-x^{j}, w^{j}-\widetilde{v}^{j}\right\rangle \leq \mu\left|y^{j}-x^{j}\right|^{2} .
$$

Then,

$$
\left|y^{j+1}-\left(x^{j}+h \widetilde{v}^{j}\right)\right|^{2} \leq\left|y^{j}-x^{j}\right|^{2}+2 h\left\langle y^{j}-x^{j}, w^{j}-\widetilde{v}^{j}\right\rangle+h^{2}\left|w^{j}-\widetilde{v}^{j}\right|^{2} .
$$

We note that the linear function $\varphi(v)=\left\langle y^{j}-x^{j}, w^{j}-v\right\rangle$ achieves its minimum on the convex compact set co $F\left(x^{j}\right)$ at some extremal point $v^{j} \in F\left(x^{j}\right)$, since the compact $F\left(x^{j}\right)$ contains all extremal points of its convex hull (see [36, Sec. III.2, Lemma 1]). Hence, we may choose $v^{j} \in F\left(x^{j}\right)$ to replace $\widetilde{v}^{j}$ in 40 .

We set $x^{j+1}:=x^{j}+h v^{j}$ and obtain from 41), 40 and (A2)

$$
\left|y^{j+1}-x^{j+1}\right|^{2} \leq(1+2 \mu h)\left|y^{j}-x^{j}\right|^{2}+4 C_{F}^{2} h^{2} .
$$

Applying (15) and Lemma 2.3, we get the estimates:

$$
\begin{aligned}
& \left|y^{j}-x^{j}\right|^{2} \leq 4 C_{F}^{2} e^{2 \mu_{+}\left(T-t_{0}\right)} \min \left\{T-t_{0}, \frac{1}{\mu}\right\} h, \\
& \left|y^{j}-x^{j}\right| \leq 2 C_{F} e^{\mu_{+}\left(T-t_{0}\right)} \min \left\{\sqrt{T-t_{0}}, \frac{1}{\sqrt{|\mu|}}\right\} \sqrt{h} .
\end{aligned}
$$

Denote by $\mathcal{S}^{c o}$ the set of solutions of the convexified differential inclusion (1) in which $F(x)$ is replaced by co $F(x)$. The following corollary extends a theorem of Grammel [22] from Lipschitz to OSL mappings $F$. 
Corollary 3.14 Under the assumptions of Theorem 3.13 and of Theorem 4.1 in [16], there is a constant $C$ such that

$$
\mathrm{d}_{\mathrm{H}}\left(\mathcal{S}_{h}, \mathcal{S}^{c o}\right) \leq C \sqrt{h} .
$$

Proof: The convergence result for the explicit Euler ([16, Theorem 4.1]) yields

$$
\mathrm{d}_{\mathrm{H}}\left(\mathcal{S}_{h}^{c o}, \mathcal{S}^{c o}\right) \leq C \sqrt{h} .
$$

The rest follows by Theorem 3.13 and the triangle inequality. $\quad$ Let us mention the conjecture of Veliov in [45] that for the Lipschitz map $F$ the above rate is $\mathcal{O}(h)$. This conjecture is proved in some important special cases.

\section{Discrete Filippov-Type Theorems for Strengthened One-Sided Lipschitz Maps}

Let us recall that the SOSL condition is stronger than the OSL, but it also provides stronger stability. In its earlier uniform version (with "for all" instead of "there exist" in its definition) in [30, 31, 32] it is implemented to gain the order of convergence 1 for the Euler method (instead of $\frac{1}{2}$ for UOSL). Several classes of discontinuous right-hand sides in applications (see [35, 38] and [25, 26, 27, 28] as well as references in [25]) fulfill the SOSL condition (see [31, 33]).

\subsection{Both perturbations}

The analysis of the convergence of the Euler scheme made in [31] and [33] lies in the basis of our proofs here. Let us stress that the uniform condition of [31, as the UOSL condition, implies uniqueness of the solution of the differential inclusion (1) which does not hold in general if the right-hand side is OSL or SOSL. In [33, Remark 2.1 and Theorem 2.4] convergence order 1 is proved for the Euler method. In the following we state discrete Filippov theorems and stability results for infinite time with estimates of order 1 improving the estimates obtained for the OSL case in Section 3 ,

Proposition 4.1 (local estimate, discrete Filippov theorem for SOSL with both perturbations)

Let the assumptions (A1)-(A2), (A3') be satisfied with the SOSL constant $\mu \in \mathbb{R}$ and choose a step size with $1+\mu h>0$.

Consider $\left\{\bar{\delta}^{j}\right\}_{j=0}^{N-1} \subset \mathbb{R}^{n}$ with $\delta_{j}:=\left|\bar{\delta}^{j}\right|_{\infty}, j=0, \ldots, N, \bar{\varepsilon}_{h}=\left\{\bar{\varepsilon}^{j}\right\}_{j=0}^{N-1} \subset \mathbb{R}^{n}, \varepsilon_{j}:=\left|\bar{\varepsilon}^{j}\right|$, $\left\{\varepsilon^{j}\right\}_{j=0}^{N-1} \subset \mathbb{R}_{+}$satisfying $\left\|\bar{\delta}_{h}\right\|_{\infty} \leq K_{\delta},\left\|\bar{\varepsilon}_{h}\right\|_{\infty} \leq K_{\varepsilon}$ and let $\left\{y^{j}\right\}_{j=0}^{N}$ be a discrete solution of the perturbed Euler inclusion

$$
y^{j+1} \in y^{j}+h\left(F\left(y^{j}+\bar{\delta}^{j}\right)+\bar{\varepsilon}^{j}\right), \quad j=0, \ldots, N-1, \quad y^{0} \in X_{0} \text { be given } .
$$

Then for every $x^{0} \in X_{0}$ there exists a discrete solution $\left\{x^{j}\right\}_{j=0}^{N}$ of (2) with

$$
\left|y^{j+1}-x^{j+1}\right|_{\infty} \leq \max \left\{(1+\mu h)\left|y^{j}-x^{j}\right|_{\infty}+|\mu| h \delta_{j}+h \varepsilon_{j}, 2 C_{F} h+\delta_{j}+h \varepsilon_{j}\right\} .
$$

Proof: By (A2), we know that all discrete Euler solutions are bounded. We denote the selections by $\left\{w^{j}\right\}_{j=0}^{N-1}$ such that

$$
y^{j+1}=y^{j}+h w^{j}+h \bar{\varepsilon}^{j}, \quad w^{j} \in F\left(y^{j}+\bar{\delta}^{j}\right) .
$$


For one iterate $y^{j} \in \mathbb{R}^{n}$ or selection $w^{j} \in \mathbb{R}^{n}$, we denote $y_{i}^{j}$ resp. $w_{i}^{j}$ as the $i$-th coordinate, where $i=1, \ldots, n$.

By assumption (A2) (the boundedness condition), the sequence $\left\{w^{j}\right\}_{j=0}^{N-1}$ is also uniformly bounded by $C_{F}$.

(i) construction of the sequence $\left\{x^{j}\right\}_{j=0}^{N-1}$

Assume that we have constructed the sequence $\left\{x^{j}\right\}_{j=0}^{k}$ up to the time step $k$. We denote the corresponding selections by $\left\{v^{j}\right\}_{j=0}^{k-1}$, i.e.,

$$
x^{j+1}=x^{j}+h v^{j}, \quad v^{j} \in F\left(x^{j}\right) .
$$

By the SOSL condition (assumption (A3')) there exists $v^{k} \in F\left(x^{k}\right)$ such that we have the SOSL inequalities as stated in (A3') in the two case: $y_{i}^{k}+\bar{\delta}_{i}^{k}>x_{i}^{k}$ (case a1 below) and $y_{i}^{k}+\bar{\delta}_{i}^{k}<x_{i}^{k}$ (case a2 below).

Other cases have to be dealt separately, e.g., case c).

(ii) local error estimate with previous error term

We set $x^{k+1}:=x^{k}+h v^{k}$ with suitable $v^{k} \in F\left(x^{k}\right)$ and consider the following cases:

case a) $\operatorname{sign}\left(\left(y_{i}^{k}+h w_{i}^{k}\right)-x_{i}^{k+1}\right)=\operatorname{sign}\left(\left(y_{i}^{k}+\bar{\delta}_{i}^{k}\right)-x_{i}^{k}\right) \neq 0$

case a1) $\left(y_{i}^{k}+h w_{i}^{k}\right)-x_{i}^{k+1}>0,\left(y_{i}^{k}+\bar{\delta}_{i}^{k}\right)-x_{i}^{k}>0$

By the SOSL condition (assumption (A3')), we find $v^{k} \in F\left(x^{k}\right)$ with

$$
w_{i}^{k}-v_{i}^{k} \leq \mu\left|\left(y^{k}+\bar{\delta}^{k}\right)-x^{k}\right|_{\infty} .
$$

In this case we distinguish two subcases, $\mu \geq 0$ and $\mu<0$. If $\mu \geq 0$, we estimate

$$
w_{i}^{k}-v_{i}^{k} \leq \mu\left|\left(y^{k}+\bar{\delta}^{k}\right)-x^{k}\right|_{\infty} \leq \mu\left|y^{k}-x^{k}\right|_{\infty}+\mu\left|\bar{\delta}^{k}\right|_{\infty} .
$$

If $\mu<0$, we use the estimate

$$
\left|\left(y^{k}-x^{k}\right)+\bar{\delta}^{k}\right|_{\infty} \geq\left|y^{k}-x^{k}\right|_{\infty}-\left|\bar{\delta}^{k}\right|_{\infty}
$$

so that

$$
w_{i}^{k}-v_{i}^{k} \leq \mu\left|\left(y^{k}+\bar{\delta}^{k}\right)-x^{k}\right|_{\infty} \leq \mu\left|y^{k}-x^{k}\right|_{\infty}-\mu\left|\bar{\delta}^{k}\right|_{\infty} .
$$

Hence, we have in both subcases the common estimate

$$
w_{i}^{k}-v_{i}^{k} \leq \mu\left|y^{k}-x^{k}\right|_{\infty}+|\mu| \cdot\left|\bar{\delta}^{k}\right|_{\infty}
$$

so that

$$
\begin{aligned}
\left|y_{i}^{k+1}-x_{i}^{k+1}\right| & \leq\left|\left(y_{i}^{k}+h w_{i}^{k}\right)-x_{i}^{k+1}\right|+h\left|\bar{\varepsilon}^{k}\right| \\
& \leq\left(\left(y_{i}^{k}+h w_{i}^{k}\right)-\left(x_{i}^{k}+h v_{i}^{k}\right)\right)+h \varepsilon_{k}=\left(y_{i}^{k}-x_{i}^{k}\right)+h\left(w_{i}^{k}-v_{i}^{k}\right)+h \varepsilon_{k} \\
& \leq\left|y_{i}^{k}-x_{i}^{k}\right|+h\left(\mu\left|y^{k}-x^{k}\right|_{\infty}+|\mu| \cdot\left|\bar{\delta}^{k}\right|_{\infty}+\varepsilon_{k}\right) \\
& \leq(1+\mu h)\left|y^{k}-x^{k}\right|_{\infty}+|\mu| h \delta_{k}+h \varepsilon_{k}
\end{aligned}
$$

a2) $\left(y_{i}^{k}+h w_{i}^{k}\right)-x_{i}^{k+1}<0,\left(y_{i}^{k}+\bar{\delta}_{i}^{k}\right)-x_{i}^{k}<0$ 
By the SOSL condition in (A3') and, as above, we have the other inequality

$$
v_{i}^{k}-w_{i}^{k} \leq \mu\left|x^{k}-y^{k}\right|_{\infty}+|\mu| \cdot\left|\bar{\delta}^{k}\right|_{\infty} .
$$

Similarly to subcase a1), we get similarly

$$
\begin{aligned}
\left|y_{i}^{k+1}-x_{i}^{k+1}\right| & \leq\left|\left(y_{i}^{k}+h w_{i}^{k}\right)-x_{i}^{k+1}\right|+h\left|\bar{\varepsilon}^{k}\right| \\
& =\left(x_{i}^{k}-y_{i}^{k}\right)+h\left(v_{i}^{k}-w_{i}^{k}\right)+h \varepsilon_{k} \\
& \leq\left|x_{i}^{k}-y_{i}^{k}\right|+h\left(\mu\left|y^{k}-x^{k}\right|_{\infty}+|\mu| \cdot\left|\bar{\delta}^{k}\right|_{\infty}+\varepsilon_{k}\right) \\
& \leq(1+\mu h)\left|y^{k}-x^{k}\right|_{\infty}+|\mu| h \delta_{k}+h \varepsilon_{k}
\end{aligned}
$$

case b) $\operatorname{sign}\left(\left(y_{i}^{k}+h w_{i}^{k}\right)-x_{i}^{k+1}\right)=-\operatorname{sign}\left(\left(y_{i}^{k}+\bar{\delta}_{i}^{k}\right)-x_{i}^{k}\right) \neq 0$

In these cases, we have an error reset, since the past estimates are not used.

b1) $\left(y_{i}^{k}+h w_{i}^{k}\right)-x_{i}^{k+1}>0,\left(y_{i}^{k}+\bar{\delta}_{i}^{k}\right)-x_{i}^{k}<0$

Here, we first proceed as in subcase a1) but do not use the SOSL condition and simply neglect negative terms:

$$
\begin{aligned}
\left|y_{i}^{k+1}-x_{i}^{k+1}\right| & \leq\left|\left(y_{i}^{k}+h w_{i}^{k}\right)-x_{i}^{k+1}\right|+h\left|\bar{\varepsilon}^{k}\right| \\
& \leq\left(y_{i}^{k}-x_{i}^{k}\right)+h\left(w_{i}^{k}-v_{i}^{k}\right)+h \varepsilon_{k} \\
& =\underbrace{\left(y_{i}^{k}+\bar{\delta}_{i}^{k}\right)-x_{i}^{k}}_{<0}+h\left(w_{i}^{k}-v_{i}^{k}\right)-\bar{\delta}_{i}^{k}+h \varepsilon_{k} \\
& <\left|\bar{\delta}_{i}^{k}\right|+h\left|w_{i}^{k}-v_{i}^{k}\right|+h \varepsilon_{k} \leq 2 C_{F} h+\delta_{k}+h \varepsilon_{k}
\end{aligned}
$$

b2) $\left(y_{i}^{k}+h w_{i}^{k}\right)-x_{i}^{k+1}<0,\left(y_{i}^{k}+\bar{\delta}_{i}^{k}\right)-x_{i}^{k}>0$

Again, we first proceed as in subcase a2) and then neglect negative terms:

$$
\begin{aligned}
\left|y_{i}^{k+1}-x_{i}^{k+1}\right| & \leq\left|\left(y_{i}^{k}+h w_{i}^{k}\right)-x_{i}^{k+1}\right|+h\left|\bar{\varepsilon}^{k}\right| \\
& =\left(x_{i}^{k}-y_{i}^{k}\right)+h\left(v_{i}^{k}-w_{i}^{k}\right)+h \varepsilon_{k} \\
& =\underbrace{x_{i}^{k}-\left(y_{i}^{k}+\bar{\delta}_{i}^{k}\right)}_{<0}+h\left(v_{i}^{k}-w_{i}^{k}\right)+\bar{\delta}_{i}^{k}+h \varepsilon_{k} \\
& <\left|\bar{\delta}_{i}^{k}\right|+h\left|w_{i}^{k}-v_{i}^{k}\right|+h \varepsilon_{k} \leq 2 C_{F} h+\delta_{k}+h \varepsilon_{k}
\end{aligned}
$$

case c) $\left(y_{i}^{k}+h w_{i}^{k}\right)-x_{i}^{k+1}=0$ or $\left(y_{i}^{k}+\bar{\delta}_{i}^{k}\right)-x_{i}^{k}=0$

c1) $\left(y_{i}^{k}+h w_{i}^{k}\right)-x_{i}^{k+1}=0$

This is the simplest case, since the essential term is zero and simply disappears.

$$
\left|y_{i}^{k+1}-x_{i}^{k+1}\right| \leq|\underbrace{\left(y_{i}^{k}+h w_{i}^{k}\right)-x_{i}^{k+1}}_{=0}|+h\left|\bar{\varepsilon}^{k}\right| \leq h \varepsilon_{k}
$$

c2) $\left(y_{i}^{k}+\bar{\delta}_{i}^{k}\right)-x_{i}^{k}=0$

Here we have

$$
\begin{aligned}
\left|y_{i}^{k+1}-x_{i}^{k+1}\right| & \leq\left|\left(y_{i}^{k}+h w_{i}^{k}\right)-x_{i}^{k+1}\right|+h\left|\bar{\varepsilon}^{k}\right| \\
& =|\underbrace{\left(y_{i}^{k}+\bar{\delta}_{i}^{k}\right)-x_{i}^{k}}_{=0}-\bar{\delta}_{i}^{k}+h\left(w_{i}^{k}-v_{i}^{k}\right)|+h \varepsilon_{k} \\
& \leq\left|\bar{\delta}_{i}^{k}\right|+h\left|w_{i}^{k}-v_{i}^{k}\right|+h \varepsilon_{k} \leq 2 C_{F} h+\delta_{k}+h \varepsilon_{k} .
\end{aligned}
$$


To summarize, in the subcases a1) and a2) we have

$$
\left|y_{i}^{k+1}-x_{i}^{k+1}\right| \leq(1+\mu h)\left|y^{k}-x^{k}\right|_{\infty}+|\mu| h \delta_{k}+h \varepsilon_{k},
$$

while in all other cases we have

$$
\left|y_{i}^{k+1}-x_{i}^{k+1}\right| \leq 2 C_{F} h+\delta_{k}+h \varepsilon_{k} .
$$

Hence, (44) holds.

We now deduce a global error estimate from the local one of Proposition 4.1. 


\section{Proposition 4.2 (global estimate, discrete Filippov theorem for SOSL with both per-} turbations)

Assume the conditions of Proposition 4.1.

Let $\left\{y^{j}\right\}_{j=0}^{N}$ be a discrete solution of the perturbed Euler inclusion

$$
y^{j+1} \in y^{j}+h\left(F\left(y^{j}+\bar{\delta}^{j}\right)+\bar{\varepsilon}^{j}\right), \quad j=0, \ldots, N-1, \quad y^{0} \in X_{0} \text { be given } .
$$

Then for every $x^{0} \in X_{0}$ there exists a discrete solution $\left\{x^{j}\right\}_{j=0}^{N}$ of (2) with

$$
\begin{aligned}
\left|y^{j}-x^{j}\right|_{\infty} \leq & \max \left\{(1+\mu h)^{j}\left|y^{0}-x^{0}\right|_{\infty},(1+\mu h)^{j}\left(2 C_{F} h+\left\|\delta_{h}\right\|_{\infty}\right)+g_{h}\left(\mu, j, \varepsilon_{h}\right)\right\} \\
& +|\mu| g_{h}\left(\mu, j, \delta_{h}\right)+g_{h}\left(\mu, j, \varepsilon_{h}\right)
\end{aligned}
$$

for $j=0, \ldots, N$ and a step size $h>0$ with $1+\mu h>0$.

Proof: The sequence $\left\{x^{j}\right\}_{j=0}^{N}$ is constructed as in Proposition 4.1.

We consider an index set $J_{\Delta} \subset\{0, \ldots, N-1\}$ for which the following holds:

- $J_{\Delta}$ consists of subsequent numbers, i.e., there are $k^{\prime} \leq k^{\prime \prime}, k^{\prime}, k^{\prime \prime} \in J_{\Delta}$ such that

$$
J_{\Delta}=\left\{k^{\prime}, k^{\prime}+1, \ldots, k^{\prime \prime}\right\}
$$

- for all indices $k \in J_{\Delta}$ we require that case a) in Proposition 4.1 holds,

- the index set $J_{\Delta}$ is maximal with respect to inclusion within the set of numbers $\{0, \ldots, N\}$.

Whenever $k \in J_{\Delta}$, we never encounter the estimate (46) and can use 45. Hence, it follows by Remark 2.2 that

$$
\left|y_{i}^{k}-x_{i}^{k}\right| \leq(1+\mu h)^{k-k^{\prime}}\left|y^{k^{\prime}}-x^{k^{\prime}}\right|_{\infty}+h \sum_{\nu=k^{\prime}}^{k-1}(1+\mu h)^{k-1-\nu}\left(|\mu| \cdot \delta_{\nu}+\varepsilon_{\nu}\right) .
$$

If this index set $J_{\Delta}$ contains the element $k^{\prime}=0$, we can rewrite the term $\left|y^{k^{\prime}}-x^{k^{\prime}}\right|_{\infty}$ as $\left|y^{0}-x^{0}\right|_{\infty}$ and we have the estimate

$$
\left|y^{k}-x^{k}\right|_{\infty} \leq(1+\mu h)^{k}\left|y^{0}-x^{0}\right|_{\infty}+|\mu| g_{h}\left(\mu, k, \delta_{h}\right)+g_{h}\left(\mu, k, \varepsilon_{h}\right) .
$$

If otherwise $k^{\prime}>0$, then we encounter an error reset due to 46 so that the maximality of $J_{\Delta}$ yields with a suitable index $i_{0} \in\{1, \ldots, n\}$ :

$$
\left|y^{k^{\prime}}-x^{k^{\prime}}\right|_{\infty}=\left|y_{i_{0}}^{k^{\prime}}-x_{i_{0}}^{k^{\prime}}\right| \leq 2 C_{F} h+\delta_{k^{\prime}-1}+h \varepsilon_{k^{\prime}-1}
$$

Thus, if $k^{\prime}>0$, we have for $k^{\prime} \leq k \leq k^{\prime \prime}$

$$
\begin{aligned}
\left|y^{k}-x^{k}\right|_{\infty} \leq & (1+\mu h)^{k-k^{\prime}}\left(2 C_{F} h+\delta_{k^{\prime}-1}+h \varepsilon_{k^{\prime}-1}\right) \\
& +|\mu| g_{h}\left(\mu, k-k^{\prime},\left\{\delta_{\nu}\right\}_{\nu=k^{\prime}}^{k-1}\right)+g_{h}\left(\mu, k-k^{\prime},\left\{\varepsilon_{\nu}\right\}_{\nu=k^{\prime}}^{k-1}\right) \\
\leq & (1+\mu h)^{k-k^{\prime}}\left(2 C_{F} h+\delta_{k^{\prime}-1}\right)+h(1+\mu h)^{k-k^{\prime}} \varepsilon_{k^{\prime}-1} \\
& +|\mu| g_{h}\left(\mu, k-k^{\prime},\left\{\delta_{\nu}\right\}_{\nu=k^{\prime}}^{k-1}\right)+g_{h}\left(\mu, k-k^{\prime},\left\{\varepsilon_{\nu}\right\}_{\nu=k^{\prime}}^{k-1}\right)
\end{aligned}
$$


Thus, we obtain in case $k^{\prime}>0, k^{\prime} \leq k \leq k^{\prime \prime}$,

$$
\begin{aligned}
\left|y^{k}-x^{k}\right|_{\infty} \leq & (1+\mu h)^{k}\left(2 C_{F} h+\left\|\delta_{h}\right\|_{\infty}\right)+g_{h}\left(\mu, k-k^{\prime},\left\{\varepsilon_{\nu}\right\}_{\nu=k^{\prime}-1}^{k-2}\right) \\
& +|\mu| g_{h}\left(\mu, k-k^{\prime},\left\{\delta_{\nu}\right\}_{\nu=k^{\prime}}^{k-1}\right)+g_{h}\left(\mu, k-k^{\prime},\left\{\varepsilon_{\nu}\right\}_{\nu=k^{\prime}}^{k-1}\right) \\
\leq & (1+\mu h)^{k}\left(2 C_{F} h+\left\|\delta_{h}\right\|_{\infty}\right)+g_{h}\left(\mu, k, \varepsilon_{h}\right) \\
& +|\mu| g_{h}\left(\mu, k, \delta_{h}\right)+g_{h}\left(\mu, k, \varepsilon_{h}\right) .
\end{aligned}
$$

The maximum of the estimates (49)-(50) implies (48).

An immediate consequence is the convergence order 1 with respect to the step size, if both perturbations are $\mathcal{O}(h)$ (measured in different norms).

Theorem 4.3 Assume the conditions of Proposition 4.2. Then,

$$
\begin{aligned}
\max _{j=0, \ldots, N}\left|y^{j}-x^{j}\right| \leq e^{\mu_{+}\left(T-t_{0}\right)}( & \max \left\{\left|y^{0}-x^{0}\right|_{\infty}, 2 C_{F} h+\left\|\delta_{h}\right\|_{\infty}+\left\lfloor\left\lfloor\varepsilon_{h}\right\rfloor_{\min , \mu}\right\}\right. \\
& +|\mu| \min \left\{T-t_{0}, \frac{1}{|\mu|}\right\}\left\|\delta_{h}\right\|_{\infty}+\left\lfloor\left\lfloor\varepsilon_{h}\right\rfloor_{\min , \mu}\right) .
\end{aligned}
$$

If $\max \left\{\left|y^{0}-x^{0}\right|_{\infty},\left\|\delta_{h}\right\|_{\infty},\left\|\varepsilon_{h}\right\|_{1}\right\} \leq C_{e} h$ also holds, then there is a constant $C$ with

$$
\left|y^{j}-x^{j}\right| \leq C h, \quad j=0, \ldots, N .
$$

A similar result as in Theorem 3.5 with $\mathcal{O}(h)$-perturbations holds for infinite time in the SOSL case.

Theorem 4.4 Assume the conditions of Proposition 4.2 on $I=\left[t_{0}, \infty\right)$ especially that $\left\|\bar{\delta}_{h}^{\infty}\right\|_{\infty} \leq$ $K_{\delta}$ and $\left\|\bar{\varepsilon}_{h}^{\infty}\right\|_{\infty} \leq K_{\varepsilon}$, and let the OSL constant satisfy $\mu<0$. For a fixed step size $h>0$ with $1+\mu h>0$ we consider infinitely many steps with the Euler method.

Then, for a discrete solution $y_{h}^{\infty}=\left\{y^{j}\right\}_{j=0}^{\infty}$ of the perturbed Euler inclusion (47) there exists a discrete solution $x_{h}^{\infty}=\left\{x^{j}\right\}_{j=0}^{\infty}$ of the Euler inclusion (2) with

$$
\begin{aligned}
\left|y^{j}-x^{j}\right| \leq & \max \left\{e^{\mu\left(t_{j}-t_{0}\right)}\left|y^{0}-x^{0}\right|_{\infty}, e^{\mu\left(t_{j}-t_{0}\right)}\left(2 C_{F} h+\left\|\left\{\delta_{\nu}\right\}_{\nu=0}^{j-1}\right\|_{\infty}\right)\right. \\
& \left.+e^{\mu_{+}\left(t_{j}-t_{0}\right)}\left\|\left\{\varepsilon_{\nu}\right\}_{\nu=0}^{j-1}\right\|_{1}\right\} \\
& +e^{\mu_{+}\left(t_{j}-t_{0}\right)}\left(\left\|\left\{\delta_{\nu}\right\}_{\nu=0}^{j-1}\right\|_{\infty}+\left\|\left\{\varepsilon_{\nu}\right\}_{\nu=0}^{j-1}\right\|_{1}\right) .
\end{aligned}
$$

Hence, the Hausdorff distance between the original and the perturbed reachable sets satisfies

$$
\limsup _{j \rightarrow \infty} \mathrm{d}_{\mathrm{H}}\left(\mathcal{R}_{h}\left(t_{j}, t_{0}, X_{0}\right), \mathcal{R}_{h}^{\varepsilon_{h}}\left(t_{j}, t_{0}, X_{0}\right)\right) \leq 2\left(\left\|\delta_{h}^{\infty}\right\|_{\infty}+\left\|\varepsilon_{h}^{\infty}\right\|_{1}\right) .
$$

Thus, if $\max \left\{\left\|\delta_{h}^{\infty}\right\|_{\infty},\left\|\varepsilon_{h}^{\infty}\right\|_{1}\right\} \leq C_{e} h$, the estimate is

$$
\limsup _{j \rightarrow \infty} \mathrm{d}_{\mathrm{H}}\left(\mathcal{R}_{h}\left(t_{j}, t_{0}, X_{0}\right), \mathcal{R}_{h}^{\varepsilon_{h}}\left(t_{j}, t_{0}, X_{0}\right)\right) \leq 4 C_{e} h
$$

where we have used Lemma 2.3 and the inequalities

$$
|\mu| e^{\mu_{+}\left(t_{j}-t_{0}\right)}\left\lfloor\left[\delta_{h}^{\infty}\right\rfloor_{\min , \mu} \leq\left\|\delta_{h}^{\infty}\right\|_{\infty}, \quad e^{\mu_{+}\left(t_{j}-t_{0}\right)}\left\lfloor\varepsilon_{h}^{\infty}\right\rfloor_{\min , \mu} \leq\left\|\varepsilon_{h}^{\infty}\right\|_{1} .\right.
$$




\subsection{Application}

For SOSL right-hand side and for the implicit Euler method we show an analogous result to Proposition 3.9, but with first order estimate replacing the $\mathcal{O}(\sqrt{h})$ order in the OSL case.

Proposition 4.5 Let the step size $h$ be so small that $h C_{F} \leq K_{\delta}, 1+2 \mu h>0$ and the assumptions (A0)-(A1), (A1'), (A3') as well as (A2) also for the implicit Euler method hold.

Then for all $x^{0} \in X_{0}$ there exists a constant $C$ such that for each iterate $\left\{y^{j}\right\}_{j=0}^{N}$ of the implicit Euler scheme

$$
y^{j+1} \in y^{j}+h F\left(y^{j+1}\right), \quad j=0,1, \ldots, N-1, \quad y^{0}=x^{0}
$$

there is one iterate $\left\{x^{j}\right\}_{j=0}^{N}$ of the explicit scheme (2) with

$$
\left|y^{j}-x^{j}\right| \leq C h, \quad j=0, \ldots, N .
$$

The distance from the reachable set of the implicit Euler method to the one of the explicit Euler and to the reachable set of (1) respectively, can be estimated by

$$
\begin{aligned}
\operatorname{dist}\left(\mathcal{R}_{h}^{i m p l}\left(T, t_{0}, X_{0}\right), \mathcal{R}_{h}\left(T, t_{0}, X_{0}\right)\right) & \leq C h, \\
\operatorname{dist}\left(\mathcal{R}_{h}^{i m p l}\left(T, t_{0}, X_{0}\right), \mathcal{R}\left(T, t_{0}, X_{0}\right)\right) & \leq C h .
\end{aligned}
$$

Proof: The proof is almost identical to the one of Proposition 3.9 and Corollary 3.10. Only [33, Theorem 2.4] and Theorem 4.3 replace [16, Theorem 4.1] and Corollary 3.7 which guarantee

$$
\left|y^{j}-x^{j}\right| \leq C h \text {. }
$$

This result is similar to the convergence order $\mathcal{O}(h)$ attained for the explicit Euler scheme in [31, but the SOSL property for the right-hand side replaces the S-UOSL property. Also the implicit Euler converges with $\mathcal{O}(h)$, if $F$ is S-UOSL.

Corollary 4.6 Let all assumptions of Proposition 4.5 hold except that (A3') is replaced by the $S$-UOSL property of $F$, then all implicit Euler iterates converges to the (single) solution of (1) with convergence order $\mathcal{O}(h)$.

Similarly, if all assumptions of Proposition 3.9 hold except that (A3) is replaced by the UOSL property of $F$, then the convergence order for the same method to the (single) solution of (1) is $\mathcal{O}(\sqrt{h})$ by [16, Theorem 4.1].

Proof: The UOSL property enforces the uniqueness of the solution $x(\cdot)$ starting from a given point such that Proposition 4.5 yields

$$
\begin{aligned}
& \operatorname{dist}\left(\mathcal{R}_{h}^{\text {impl }}\left(T, t_{0},\left\{x_{0}\right\}\right),\{x(T)\}\right) \leq C h, \\
\operatorname{dist}\left(\{x(T)\}, \mathcal{R}_{h}^{\text {impl }}\left(T, t_{0},\left\{x_{0}\right\}\right)\right)= & \inf _{\eta^{N} \in \mathcal{R}_{h}^{\text {impl }}\left(T, t_{0},\left\{x_{0}\right\}\right)}\left|x(T)-\eta^{N}\right| \\
\leq & \sup _{\eta^{N} \in \mathcal{R}_{h}^{\text {impl }}\left(T, t_{0},\left\{x_{0}\right\}\right)}\left|x(T)-\eta^{N}\right|=\operatorname{dist}\left(\mathcal{R}_{h}^{\text {impl }}\left(T, t_{0},\left\{x_{0}\right\}\right),\{x(T)\}\right) \leq C h .
\end{aligned}
$$

Remark 4.7 The same $\mathcal{O}(h)$-estimate may also be obtained for the improved Euler (cp. Remark 3.11) in the case if $F$ is SOSL.

Acknowledgements: The authors would like to thank Frank Lempio and Janosch Rieger for the helpful discussions on the explicit and implicit Euler method. The authors also acknowledge the partial support by The Hermann Minkowski Center for Geometry at Tel Aviv University, Israel. 


\section{References}

[1] J.-P. Aubin and A. Cellina. Differential Inclusions, volume 264 of Grundlehren der mathematischen Wissenschaften. Springer-Verlag, Berlin-Heidelberg-New York-Tokyo, 1984.

[2] J.-P. Aubin and H. Frankowska. Set-Valued Analysis, volume 2 of Systems 8 Control: Foundations \& Applications. Birkhäuser Boston Inc., Boston, MA, 1990.

[3] W. Auzinger, R. Frank, and F. Macsek. Asymptotic error expansions for stiff equations: the implicit Euler scheme. SIAM J. Numer. Anal., 27(1):67-104, 1990.

[4] R. Baier, I. A. Chahma, and F. Lempio. Stability and convergence of Euler's method for state-constrained differential inclusions. SIAM J. Optim., 18(3):1004-1026 (electronic), 2007. D. Dentcheva, J. Revalski (eds.), special issue on "Variational Analysis and Optimization".

[5] R. Baier and E. Farkhi. Regularity of set-valued maps and their selections through set differences. Part 2: One-sided Lipschitz properties. Serdica Math. J., 39(3-4):391-422, 2013. Special issue dedicated to the 65th anniversary of Professor Asen L. Dontchev and to the 60th anniversary of Professor Vladimir M. Veliov.

[6] W.-J. Beyn and J. Rieger. The implicit Euler scheme for one-sided Lipschitz differential inclusions. Dyn. Contin. Discrete Impuls. Syst. Ser. B Appl. Algorithms, 14(2):409-428, 2010.

[7] F. E. Browder. Nonlinear accretive operators in Banach spaces. Bull. Amer. Math. Soc., 73:470-476, 1967.

[8] F. E. Browder. Nonlinear mappings of nonexpansive and accretive type in Banach spaces. Bull. Amer. Math. Soc., 73:875-882, 1967.

[9] I. A. Chahma. Set-valued discrete approximation of state-constrained differential inclusions. Bayreuth. Math. Schr., 67:3-162, 2003.

[10] K. Deimling. Multivalued Differential Equations, volume 1 of de Gruyter Series in Nonlinear Analysis and Applications. Walter de Gruyter, Berlin-New York, 1992.

[11] K. Dekker and J. G. Verwer. Stability of Runge-Kutta methods for stiff nonlinear differential equations, volume 2 of CWI Monographs. North-Holland Publishing Co., Amsterdam, 1984.

[12] T. D. Donchev. Functional-differential inclusion with monotone right-hand side. Nonlinear Anal., 16(6):533-542, 1991.

[13] T. D. Donchev. Properties of one-sided Lipschitz multivalued maps. Nonlinear Anal., 49(1):13$20,2002$.

[14] T. D. Donchev. One sided Lipschitz multifunctions and applications. In Optimal Control, Stabilization and Nonsmooth Analysis, volume 301 of Lecture Notes in Control and Inform. Sci., pages 333-341. Springer, Berlin, 2004.

[15] T. D. Donchev and E. Farkhi. Stability and Euler approximation of one-sided Lipschitz differential inclusions. SIAM J. Control Optim., 36(2):780-796 (electronic), 1998. 
[16] T. D. Donchev and E. Farkhi. Approximations of one-sided Lipschitz differential inclusions with discontinuous right-hand sides. In Calculus of Variations and Differential Equations (Haifa, 1998), volume 410 of Chapman $\&$ Hall/CRC Res. Notes Math., pages 101-118. Chapman \& Hall/CRC, Boca Raton, FL, 2000.

[17] T. D. Donchev and E. Farkhi. On the theorem of Filippov-Pliś and some applications. Control and Cybernet., 38(4A):1251-1271, 2009.

[18] T. D. Donchev and R. Ivanov. On the existence of solutions of differential inclusions in uniformly convex Banach space. Math. Balkanica (N.S.), 6(1):13-24, 1992.

[19] E. Farkhi, T. D. Donchev, and R. Baier. Existence of solutions for nonconvex differential inclusions of monotone type. C. R. Acad. Bulgare Sci., 67(3):323-330, 2014.

[20] A. F. Filippov. Classical solutions of differential equations with multi-valued right-hand side. SIAM J. Control, 5:609-621, 1967.

[21] H. Frankowska and F. Rampazzo. Filippov's and Filippov-Wazewski's theorems on closed domains. J. Differ. Equ., 161(2):449-478, 2000.

[22] G. Grammel. Towards fully discretized differential inclusions. Set-Valued Anal., 11(1):1-8, 2003.

[23] E. Hairer and G. Wanner. Solving Ordinary Differential Equations. II Stiff and DifferentialAlgebraic Problems, volume 14 of Springer Series in Computational Mathematics. SpringerVerlag, Berlin, second edition, 1996.

[24] J. L. Haunschmied, A. Pietrus, and V. M. Veliov. The Euler method for linear control systems revisited. In I. Lirkov, S. D. Margenov, and J. Waśniewski, editors, Large-Scale Scientific Computing. Revised selected papers from the 9th Internat. Conf. (LSSC '13), Sozopol, Bulgaria, June 3-7, 2013, volume 8353 of Lecture Notes in Comput. Sci., pages 90-97. Springer, Heidelberg, 2014.

[25] A. Kastner-Maresch. Diskretisierungsverfahren zur Lösung von Differentialinklusionen [Discretization Methods for the Solution of Differential Inclusions]. PhD thesis, Department of Mathematics, University of Bayreuth, Bayreuth, Germany, January 1990.

[26] A. Kastner-Maresch. Implicit Runge-Kutta methods for differential inclusions. Numer. Funct. Anal. Optim., 11(9-10):937-958 (1991), 1990.

[27] A. Kastner-Maresch. The implicit midpoint rule applied to discontinuous differential equations. Computing, 49(1):45-62, 1992.

[28] A. Kastner-Maresch and F. Lempio. Difference methods with selection strategies for differential inclusions. Numer. Funct. Anal. Optim., 14(5-6):555-572, 1993.

[29] F. Lempio. Difference methods for differential inclusions. In Modern Methods of Optimization. Proceedings of a Summer School at the Schloß Thurnau of the University of Bayreuth (Germany), FRG, October 1-6, 1990, volume 378 of Lecture Notes in Econom. and Math. Systems, pages 236-273. Springer-Verlag, Berlin-Heidelberg-New York, 1992. 
[30] F. Lempio. Modified Euler methods for differential inclusions. In Set-Valued Analysis and Differential Inclusions. A Collection of Papers resulting from a Workshop held in Pamporovo, Bulgaria, September 17-21, 1990, volume 16 of Progr. Systems Control Theory, pages 131-148. Birkhäuser Boston, Boston, MA-Basel-Berlin, 1993.

[31] F. Lempio. Euler's method revisited. Proc. Steklov Inst. Math., 211:429-449, 1995.

[32] F. Lempio and D. B. Silin. Differential inclusions with strongly one-sided-Lipschitz right-hand sides. Differ. Equ., 32(11):1485-1491, 1997.

[33] F. Lempio and V. M. Veliov. Discrete approximations of differential inclusions. Bayreuth. Math. Schr., 54:149-232, 1998.

[34] G. Lumer and R. S. Phillips. Dissipative operators in a Banach space. Pacific J. Math., 11:679-698, 1961.

[35] R. Mannshardt. One-step methods of any order for ordinary differential equations with discontinuous right-hand sides. Numer. Math., 31(2):131-152, 1978/79.

[36] J. T. Marti. Konvexe Analysis, volume 54 of Lehrbücher und Monographien aus dem Gebiet der Exakten Wissenschaften, Mathematische Reihe. Birkhäuser, Basel-Stuttgart, 1977.

[37] R. H. Martin, Jr. A global existence theorem for autonomous differential equations in a Banach space. Proc. Amer. Math. Soc, 26:307-314, 1970.

[38] R. Model. Zur Integration über Unstetigkeiten in gewöhnlichen Differentialgleichungen. Z. Angew. Math. Mech., 68(3):161-169, 1988.

[39] A. Pietrus and V. M. Veliov. On the discretization of switched linear systems. Systems Control Lett., 58(6):395-399, 2009.

[40] V. M. Veliov. Second order discrete approximations to strongly convex differential inclusions. Systems Control Lett., 13(3):263-269, 1989.

[41] V. M. Veliov. Second Order Discrete Approximation to Linear Differential Inclusions. SIAM J. Numer. Anal., 29(2):439-451, 1992.

[42] V. M. Veliov. On the time-discretization of control systems. SIAM J. Control Optim., $35(5): 1470-1486,1997$.

[43] V. M. Veliov. Error analysis of discrete approximations to bang-bang optimal control problems: the linear case. Control Cybernet., 34(3):967-982, 2005.

[44] V. M. Veliov. On the relationship between continuous- and discrete-time control systems. CEJOR Cent. Eur. J. Oper. Res., 18(4):511-523, 2010.

[45] V. M. Veliov. Relaxation of Euler-type discrete-time control system. In I. Lirkov, S. D. Margenov, and J. Waśniewski, editors, Large-Scale Scientific Computing. Revised selected papers from the 10th Internat. Conf. (LSSC '15), Sozopol, Bulgaria, June 8-12, 2015, volume 9374 of Lecture Notes in Comput. Sci., pages 134-141. Springer, Cham, 2015. 